\title{
Better timing for cord clamping is after onset of lung aeration
}

\author{
Satyan Lakshminrusimha' and Krisa Van Meurs
}

\begin{abstract}
$\mathrm{n}$ the Scandinavian congress of physiologists in 1959, Dr John Lind showed a film titled "Roentgen cinematographic studies on aeration of the lung at birth" (1). He demonstrated that tying the umbilical cord prior to the first breath reduces the size of the heart during the first three or four cardiac cycles. The heart again increased in size, almost to that of the fetal heart. This change in heart size was interpreted as the filling of the opened pulmonary vasculature occurring with onset of lung aeration after birth.
\end{abstract}

"If the umbilical circulation is closed, the flow from the caudal caval vein through via sinistra (umbilical venous stream flowing into the left atrium through the foramen ovale, as opposed to via dextra - flowing into the right ventricle) to the left heart will hardly suffice and for a moment the left heart will not have enough blood. On the basis of these observations, it would seem that the closing of the umbilical circulation before the aeration of the lungs has taken place is a highly 'unphysiological' measure, which should thus be avoided" (2).

This astute observation summarized by Peltonen in 1981 identified umbilical venous return as the main contributor to left ventricular preload in the fetal period. In human fetuses, a strong inverse relationship exists between foramen ovale shunt and pulmonary venous return (3). The foramen ovale shunt is primarily dependent on umbilical venous return while pulmonary venous return is the second source of left ventricular preload. This relationship enables one source of fetal left ventricular preload (increased foramen ovale shunt) to compensate for the other (decreased pulmonary blood flow with fetal hypoxia) $(4,5)$. In this dynamic manner, adequate left ventricular output and blood supply to essential organs are maintained during fetal life.

In this issue of Pediatric Research, Hooper et al. provide an excellent review of the "physiological sequence" of cardiovascular transition at birth (6). Based on elegant data from preterm lambs (7), the authors demonstrate that if ventilation is not established prior to "early" cord clamping, the left ventricle is deprived of both sources of preload (Figure 1). Increased afterload secondary to cord clamping may put additional strain on the left ventricle, and this decrease in left ventricular output has the potential to induce an ischemic insult. They suggest that cord clamping should be performed after establishing effective ventilation so that pulmonary venous return can replace umbilical venous return as the source of left ventricular preload (Figure 2). Hooper et al. argue that optimal time of cord clamping after birth should be based on infant's physiology, specifically lung aeration. It is inappropriate to set an arbitrary time for cord clamping that focuses solely on time from delivery or the volume of the placental transfusion without paying attention to other important aspects of the physiologic transition. Ersdal et al. found a similar conclusion in their study of self-breathing neonates in a low-resource setting in rural Tanzania (8). A higher risk of death and/or remaining admitted at $24 \mathrm{~h}$ was seen if cord clamping occurred before or immediately after onset of spontaneous respiration. The odds ratio for death/admission decreased by $20 \%$ for every $10 \mathrm{~s}$ delay in cord clamping after initiation of spontaneous respirations up to $2 \mathrm{~min}$. This is a powerful testament to the global impact of a physiology-based approach at delivery as outlined by Hooper et al.

The optimal time for cord clamping has been a controversial topic for several decades. In the 1950s and 60s, investigators from Sweden, the United States and Canada evaluated blood volume changes after birth. These studies demonstrated that blood volume, venous hematocrit and red blood cell volume were significantly higher when cord clamping was delayed for either 1 or $5 \mathrm{~min}$ (9). Paradoxically, subsequent response to these findings, the interval between birth and cord clamping began to be shortened and was generally performed within 15-20 s after birth (10). Delayed cord clamping is generally defined as umbilical cord clamping performed 30-60 s after birth. Neither of these "definitions" give due consideration to the physiologic status of the newborn infant. Thankfully, many infants know better and cry immediately after birth before the umbilical cord is clamped.

What are the benefits of delayed cord clamping in term and preterm infants? Studies in healthy term infants suggest $~ 70-120 \mathrm{~g}$ weight increase with a several minute delay in cord clamping $(11,12)$. This additional blood volume results in a higher hemoglobin, reduces the need for transfusion, and provides extra iron to prevent iron deficiency during infancy; however, these benefits must be weighed against the increased risk for polycythemia and need for phototherapy (13). Despite these beneficial findings, recommendations vary. The American College of Obstetrics and Gynecology in 2012 concluded

'Department of Pediatrics, University at Buffalo, Buffalo, New York; ${ }^{2}$ Division of Neonatal and Developmental Medicine, Department of Pediatrics, Stanford University School of Medicine, Palo Alto, California. Correspondence: Satyan Lakshminrusimha (slakshmi@buffalo.edu) 


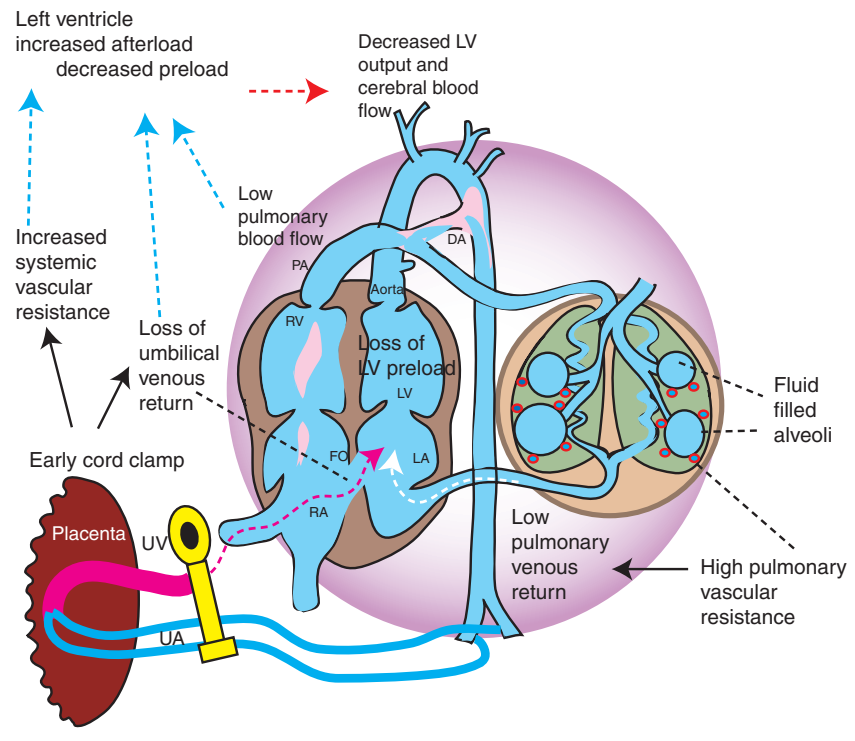

Figure 1. Early cord clamping prior to onset of lung aeration. Immediate cord clamping prior to lung aeration restricts flow to the ventricles. With failure to establish ventilation, pulmonary vascular resistance (PVR) remains high and compromises pulmonary blood flow (increased right to left DA shunt) and venous return to the left ventricle. Decreased filling of the left ventricle (preload) and increased afterload (due to removal of low-resistance placenta) compromise cardiac output. DA, ductus arteriosus; $L A$, left atrium; $L V$, left ventricle; $R A$, right atrium; $R V$, right ventricle; UA, umbilical artery; UV, umbilical vein. that in term infants there was insufficient evidence to either refute or support delaying cord clamping in settings with rich resources, but in settings with prevalent iron deficiency anemia it was warranted (10). The International Liaison Committee on Resuscitation in 2011 recommended delayed cord clamping for $1 \mathrm{~min}$ in term infants not requiring resuscitation (14). In preterm infants, there is stronger evidence of short-term benefit. Delayed cord clamping for 30-60 s in infants $<32 \mathrm{wk}$ gestation has been shown to increase blood pressure at 1 and $4 \mathrm{~h}$, lower the need for blood transfusion and reduce the incidence of any intraventricular hemorrhage and necrotizing enterocolitis (15). American College of Obstetrics and Gynecology concluded, "evidence supports delayed cord clamping in preterm infants" but they acknowledge that the ideal timing has yet to be established and timing in relation to initiation of voluntary or assisted ventilation deserves further study (10). Recent European guidelines reviewed the same body of literature and recommend: "if possible delay clamping for at least 60 seconds with the baby held below the mother to promote placentofetal transfusion" (16). In the preterm population as in the term infant, the effect of delayed cord clamping on long-term neurodevelopmental outcomes has not been adequately studied.

What are the potential risks of delayed cord clamping? The foremost concern is that timely resuscitation efforts may be postponed, especially in extremely low birth weight or ill-term infants. However, because the placenta continues to perform

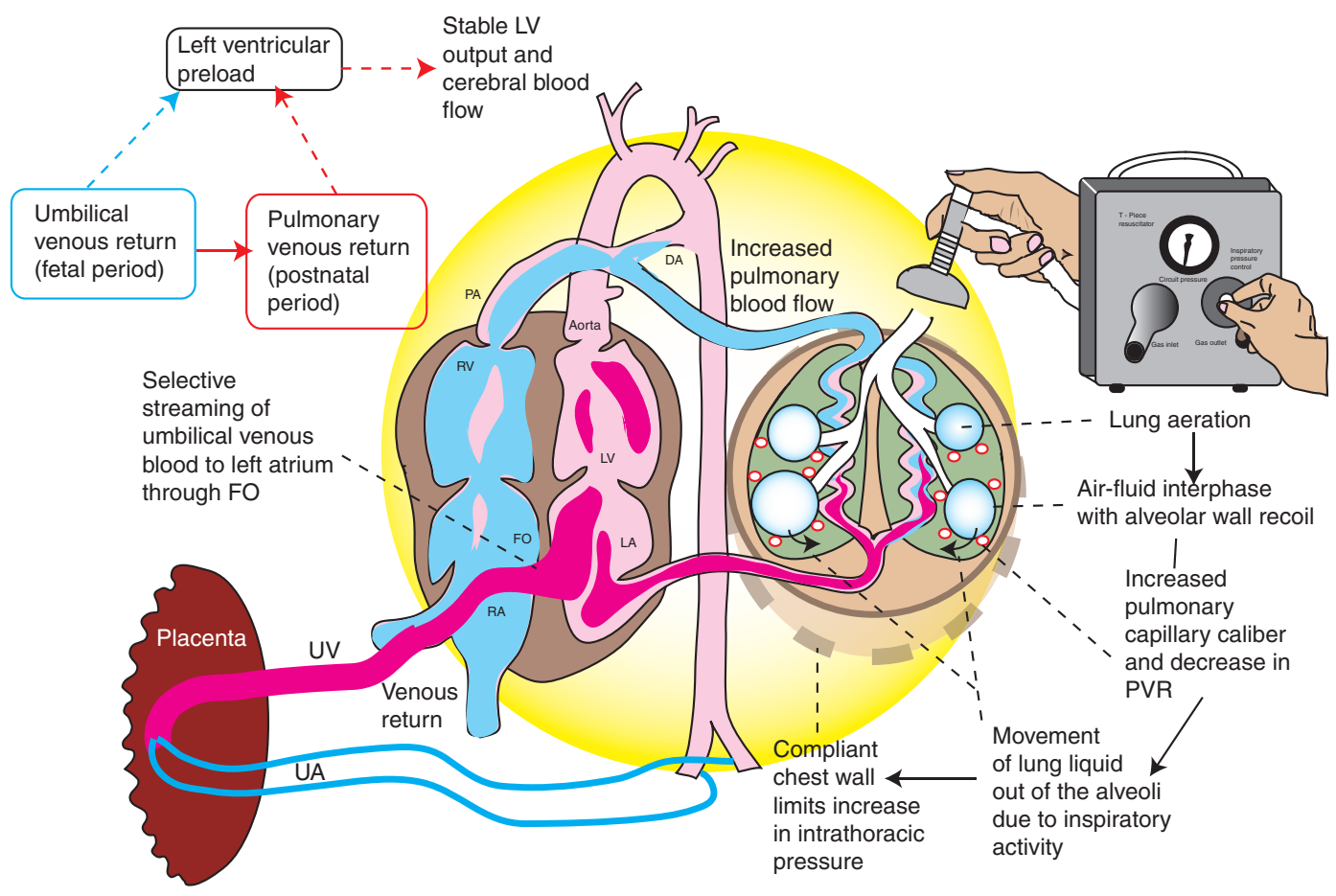

Figure 2. Physiologic (delayed) cord clamping after onset of lung aeration. The left ventricular preload occurs from two sources: umbilical venous return through the FO shunt (predominant source during fetal life) and pulmonary venous return (main source after birth). An intact umbilical cord allows continuous umbilical venous flow to the ventricles while respirations are being established. With the concomitant initiation of breathing through crying or positive pressure ventilation (PPV), pulmonary vascular resistance (PVR) decreases allowing increased blood flow to the lungs (decreased right to left shunt through the ductus arteriosus (DA)) as well as increased venous return to the LV. The unclamped UA prevents a sudden increase in left ventricular afterload. These factors result in stable cardiac output. Clearance of lung liquid and an increase in resting air volume leads to thoracic expansion in the presence of a compliant chest wall limiting the increase in intrathoracic pressure. FO, foramen ovale; LA, left atrium; LV, left ventricle; RA, right atrium; $\mathrm{RV}$, right ventricle; UA, umbilical artery; UV, umbilical vein. 
gas exchange after delivery (unless the placenta or the cord are compromised), sick-term and preterm infants are most likely to benefit from the placental transfusion. Excessive placental transfusion leading to neonatal polycythemia and possibly hyperbilirubinemia is a potential concern with delayed cord clamping; however, the hematological benefits of placental transfusion such as higher hematocrits and fewer transfusions outweigh these risks in extremely preterm infants. There remains a theoretical concern regarding the effect of delayed cord clamping on maternal hemorrhage. However, a Cochrane review performed in 2012 found no significant differences in postpartum hemorrhage between early ( $<1 \mathrm{~min}$ ) and late cord clamping (13).

How should newborn infants that are not vigorous due to prematurity, asphyxia or maternal medications or anesthesia be managed? There is inadequate data to answer this important question. Insufficient numbers of infants less than $30 \mathrm{wk}$ gestation have been enrolled in trials comparing placental transfusion to immediate cord clamping; furthermore, results and conclusions have differed regarding mortality and intraventricular hemorrhage $(17,18)$. Hooper et al. stress the importance of lung aeration prior to cord clamping. Initiation of positive pressure ventilation using a sterile mask with a T-piece device with an intact umbilical cord is an option or alternatively, the umbilical cord may be "milked" to expedite placental transfusion $(19,20)$. In the UK CORD trial, the delayed cord-clamping arm specifies resuscitation of the infant at the mother's bedside permitting the cord to remain intact in excess of $2 \mathrm{~min}$ during which time lung aeration should occur (21). A number of international clinical trials are underway in newborns less than $30-32 \mathrm{wk}$ gestation and these trials are expected to enroll several thousand newborns. Meta-analyses of trial results will provide important evidence to guide future practice. Similar trials in asphyxiated term infants are warranted.

In this issue of Pediatric Research, Hooper et al. convincingly present physiologic evidence demonstrating that the relative timing of cord clamping and lung aeration is critical to cardiovascular stability after birth. Newborns requiring resuscitation at birth are probably better off remaining attached to the umbilical cord and having ventilation initiated before the cord is clamped. This sequence prevents a reduction in ventricular preload and cardiac output caused by the simultaneous loss of umbilical venous return (early cord clamping) and low pulmonary blood flow (lack of lung aeration). Randomized controlled trials of delayed cord clamping after onset of ventilation are needed to determine convincingly whether this practice will result in short-term benefits as well as improved long-term neurodevelopmental outcomes.

\section{STATEMENT OF FINANCIAL SUPPORT}

American Academy of Pediatrics, Neonatal Resuscitation Program (SL) and 1R01HD072929-0 (SL) and Department of Pediatrics, University at Buffalo, Buffalo, NY.

\section{Disclosure: There is no conflict of interest to disclose.}

\section{REFERENCES}

1. Lind J, Peltonen T, Törnwall L, Wegelius CW. Film: Roentgen cinematographic studies of the aeration of the lung at birth. The Second Scandinavian
Summer Meeting of Biochemistry, Medical Chemistry, Pharmacology, and Physiology. Turku, 27-29 August 1959.

2. Peltonen T. Placental transfusion-advantage an disadvantage. Eur J Pediatr 1981;137:141-6.

3. Prsa M, Sun L, van Amerom J, et al. Reference ranges of blood flow in the major vessels of the normal human fetal circulation at term by phasecontrast magnetic resonance imaging. Circ Cardiovasc Imaging 2014;7: 663-70.

4. Cohn HE, Sacks EJ, Heymann MA, Rudolph AM. Cardiovascular responses to hypoxemia and acidemia in fetal lambs. Am J Obstet Gynecol 1974;120:817-24.

5. Mäkikallio K, Erkinaro T, Niemi N, et al. Fetal oxygenation and Doppler ultrasonography of cardiovascular hemodynamics in a chronic near-term sheep model. Am J Obstet Gynecol 2006;194:542-50.

6. Hooper SB, te Pas AB, Lang J, et al. The cardiovascular transition at birth: a physiological sequence. Pediatr Res 2014;77:608-614.

7. Bhatt S, Alison BJ, Wallace EM, et al. Delaying cord clamping until ventilation onset improves cardiovascular function at birth in preterm lambs. J Physiol (Lond) 2013;591(Pt 8):2113-26.

8. Ersdal HL, Linde J, Mduma E, Auestad B, Perlman J. Neonatal outcome following cord clamping after onset of spontaneous respiration. Pediatrics 2014;134:265-72.

9. Saigal S, O'Neill A, Surainder Y, Chua LB, Usher R. Placental transfusion and hyperbilirubinemia in the premature. Pediatrics 1972;49:406-19.

10. Committee on Obstetric Practice, American College of Obstetricians and Gynecologists. Committee Opinion No. 543: Timing of umbilical cord clamping after birth. Obstet Gynecol 2012;120:1522-6.

11. Farrar D, Airey R, Law GR, Tuffnell D, Cattle B, Duley L. Measuring placental transfusion for term births: weighing babies with cord intact. BJOG 2011;118:70-5.

12. Hutton EK, Hassan ES. Late vs early clamping of the umbilical cord in fullterm neonates: systematic review and meta-analysis of controlled trials. JAMA 2007;297:1241-52.

13. McDonald SJ, Middleton P, Dowswell T, Morris PS. Effect of timing of umbilical cord clamping of term infants on maternal and neonatal outcomes. Cochrane Database Syst Rev 2013;7:CD004074.

14. Perlman JM, Wyllie J, Kattwinkel J, et al.; Neonatal Resuscitation Chapter Collaborators. Part 11: Neonatal resuscitation: 2010 International Consensus on Cardiopulmonary Resuscitation and Emergency Cardiovascular Care Science With Treatment Recommendations. Circulation 2010;122(16 Suppl 2):S516-38.

15. Rabe H, Diaz-Rosello JL, Duley L, Dowswell T. Effect of timing of umbilical cord clamping and other strategies to influence placental transfusion at preterm birth on maternal and infant outcomes. Cochrane Database Syst Rev 2012;9:CD003248.

16. Sweet DG, Carnielli V, Greisen G, et al.; European Association of Perinatal Medicine. European consensus guidelines on the management of neonatal respiratory distress syndrome in preterm infants-2013 update. Neonatology 2013;103:353-68.

17. Ghavam S, Batra D, Mercer J, et al. Effects of placental transfusion in extremely low birthweight infants: meta-analysis of long- and short-term outcomes. Transfusion 2014;54:1192-8.

18. Backes $\mathrm{CH}$, Rivera BK, Haque U, et al. Placental transfusion strategies in very preterm neonates: a systematic review and meta-analysis. Obstet Gynecol 2014;124:47-56.

19. Hosono S, Mugishima H, Fujita H, et al. Umbilical cord milking reduces the need for red cell transfusions and improves neonatal adaptation in infants born at less than 29 weeks' gestation: a randomised controlled trial. Arch Dis Child Fetal Neonatal Ed 2008;93:F14-9.

20. Rabe H, Jewison A, Alvarez RF, et al.; Brighton Perinatal Study Group. Milking compared with delayed cord clamping to increase placental transfusion in preterm neonates: a randomized controlled trial. Obstet Gynecol 2011;117(2 Pt 1):205-11.

21. Pushpa-Rajah A, Bradshaw L, Dorling J, et al.; Cord Pilot Trial Collaborative Group. Cord pilot trial - immediate versus deferred cord clamping for very preterm birth (before 32 weeks gestation): study protocol for a randomized controlled trial. Trials 2014;15:258. 